\title{
HYPERBOLIC SPACES AND QUADRATIC FORMS
}

\author{
JOSEPH E. VALENTINE
}

1. Introduction. A semimetric $(n+1)$-tuple is a set of $n+1$ "points" $p_{0}, p_{1}, \cdots, p_{n}$ with a nonnegative real number $p_{i} p_{j}=p_{j} p_{i}(i, j$ $=0,1, \cdots, n)$, attached to each pair of elements, while $p_{i} p_{j}=0$ if and only if $i=j$; i.e., if and only if $p_{i}=p_{j}$. Coxeter and Todd [4] have even investigated spaces with distances not necessarily real numbers. Menger [5], [6] investigated necessary and sufficient conditions that a semimetric $(n+1)$-tuple be isometrically imbeddable in a euclidean $n$-dimensional space $E_{n}$. He solved this problem by means of equations and inequalities involving certain determinants. Blumenthal and Garrett [3] and Blumenthal [2] solved the similar problems for $n$-dimensional spherical space $S_{n, r}$ of radius $r$ and $n$-dimensional hyperbolic space $H_{n, r}$ of curvature $-1 / r^{2}, r>0$ by means of certain determinants. Schoenberg [7], [8] gave complete and independent solutions for the problems for euclidean and spherical spaces by means of quadratic forms. The purpose of this paper is to solve the problem for hyperbolic space by means of quadratic forms. A related problem in hyperbolic space is solved. The solution to this problem is quite trivial in the setting of quadratic forms, while it would be quite difficult if only the determinant conditions of Blumenthal were available.

2. The criterion. A complete solution to the problem of necessary and sufficient conditions that a semimetric $(n+1)$-tuple be isometrically imbeddable in hyperbolic space is given by the following theorem.

Theorem. A necessary and sufficient condition that a semimetric $(n+1)$-tuple, $p_{0}, p_{1}, \cdots, p_{n}$ be congruently imbeddable in $H_{k, r}$ and not in $H_{k-1, r}$ is that the quadratic form

$G\left(x_{1}, x_{2}, \cdots, x_{n}\right)=\sum_{i, j=1}^{n}\left[\cosh \left(p_{0} p_{i} / r\right) \cosh \left(p_{0} p_{j} / r\right)-\cosh \left(p_{i} p_{j} / r\right)\right] x_{i} x_{j}$

be positive semidefinite of rank $k$.

Proof. Suppose points $q_{0}, q_{1}, \cdots, q_{n}$ of $H_{n, \text { r }}$ exist such that $p_{i} p_{j}$ $=q_{i} q_{j}(i, j=0,1,2, \cdots n)$. It is known, [1, Exercise 2, p. 273], that the " $n$-bein" formed by the rays

Received by the editors May 1, 1969. 


$$
\overrightarrow{q_{0} q_{h}} \quad(h=1,2, \cdots, n)
$$

of $H_{n, r}$ is isogonally imbeddable in an $n$-dimensional euclidean space $E_{n}$. Let $\mu$ denote the " $n$-bein" of $E_{n}$ which is isogonal with the " $n$-bein" of $H_{n, r}$, and let $\rho_{h}$ correspond to

$$
\overrightarrow{q_{0} q_{h}} \quad(h=1,2, \cdots, n) .
$$

If $\rho_{h}$ intersects the unit sphere $S_{n-1,1}$ with center at the vertex of $\mu$ in $p_{h}^{\prime}(h=1,2, \cdots, n)$, then $p_{i}^{\prime} p_{j}^{\prime}=\operatorname{spher} \operatorname{dist}\left(p_{i}^{\prime}, p_{j}^{\prime}\right)=\Varangle q_{0}: q_{i}, q_{j}$ $(i, j=1,2, \cdots, n)$, where $\Varangle q_{0}: q_{i}, q_{j}$ denotes the angle formed at $q_{0}$ by the rays

$$
\overrightarrow{q_{0} q_{i}}, \quad \vec{q}_{0} q_{j}
$$

By the hyperbolic law of cosines,

$$
\begin{aligned}
\cos \left(\chi_{4} q_{0}: q_{i}, q_{j}\right)= & {\left[\cosh \left(q_{0} q_{i} / r\right) \cosh \left(q_{0} q_{j} / r\right)\right.} \\
& \left.-\cosh \left(q_{i} q_{j} / r\right)\right] /\left[\sinh \left(q_{0} q_{i} / r\right) \sinh \left(q_{0} q_{j} / r\right)\right]
\end{aligned}
$$

thus,

$$
\begin{aligned}
\cos \left(p_{i}{ }^{\prime} p_{j}{ }^{\prime}\right)= & {\left[\cosh \left(q_{0} q_{i} / r\right) \cosh \left(q_{0} q_{j} / r\right)\right.} \\
& \left.-\cosh \left(q_{i} q_{j} / r\right)\right] /\left[\sinh \left(q_{0} q_{i} / r\right) \sinh \left(q_{0} q_{j} / r\right)\right] .
\end{aligned}
$$

According to Theorem $2[6$, pp. 727, 728] a necessary and sufficient condition that points $p_{i}^{\prime}, p_{j}^{\prime}(i, j=1,2, \cdots, n)$ exist on the unit sphere $S_{n-1,1}$ is that the quadratic form $\Phi\left(x_{1}, x_{2}, \cdots, x_{n}\right)$ $=\sum_{i, j=1}^{n} \cos \left(p_{i}^{\prime} p_{j}^{\prime}\right) x_{i} x_{j}$ be positive semidefinite. Moreover, if the rank of $\Phi$ is $k$, then such points lie on $S_{k-1,1}$ but not on $S_{k-2,1}$.

Hence, a necessary and sufficient condition that a semimetric $(n+1)$-tuple $p_{0}, p_{1}, \cdots, p_{n}$ be isometrically imbeddable in $H_{k, r}$ and not in $H_{k-1, r}$ is that the quadratic form

$$
\begin{aligned}
G^{\prime}\left(x_{1}, x_{2}, \cdots, x_{n}\right)= & \sum_{i, j=1}^{n}\left[\cosh \left(p_{0} p_{i} / r\right) \cosh \left(p_{0} p_{j} / r\right)\right. \\
& \left.-\cosh \left(p_{i} p_{j} / r\right)\right] /\left[\sinh \left(p_{0} p_{i} / r\right) \sinh \left(p_{0} p_{j} / r\right)\right] x_{i} x_{j}
\end{aligned}
$$

be positive semidefinite of rank $k$.

Now $G^{\prime}$ is positive semidefinite of rank $k$ if and only if the quadratic form

$$
G\left(x_{1}, x_{2}, \cdots, x_{n}\right)=\sum_{i, j=1}^{n}\left[\cosh \left(p_{0} p_{i} / r\right) \cosh \left(p_{0} p_{j} / r\right)-\cosh \left(p_{i} p_{j} / r\right)\right] x_{i} x_{j}
$$


has the same properties.

Corollary. A semimetric $(n+1)$-tuple $p_{0}, p_{1}, \cdots, p_{n}$ is isometrically imbeddable in $H_{k, r}$ and not in $H_{k-1, r}$ if and only if the quadratic form $\sum_{i, j=0}^{n} \cosh \left(p_{i} p_{j} / r\right) x_{i} x_{j}$ is negative semidefinite of rank $k$ on the hyperplane $x_{0}=-\sum_{j=1}^{n} \cosh \left(p_{0} p_{j} / r\right) x_{j}$.

Proof. It is clear that

$$
\begin{aligned}
\sum_{i, j=0}^{n} \cosh \left(p_{i} p_{j} / r\right) x_{i} x_{j}= & x_{0}^{2}+2 x_{0} \sum_{i=1}^{n} \cosh \left(p_{0} p_{i} / r\right) x_{i} \\
& +\sum_{i, j=1}^{n} \cosh \left(p_{i} p_{j} / r\right) x_{i} x_{j} ;
\end{aligned}
$$

and for all values of $x_{0}, x_{1}, \cdots, x_{n}$ such that $x_{0}=-\sum_{j=1}^{n} \cosh \left(p_{0} p_{j} / r\right) x_{j}$ we have

$$
\begin{aligned}
\sum_{i, j=0}^{n} \cosh \left(p_{i} p_{j} / r\right) x_{i} x_{j} \\
\quad=-\sum_{j=1}^{n} \cosh \left(p_{0} p_{j} / r\right) x_{j} \sum_{i=1}^{n} \cosh \left(p_{0} p_{i} / r\right) x_{i}+\sum_{i, j=1}^{n} \cosh \left(p_{i} p_{j} / r\right) x_{i} x_{j} \\
=-\sum_{i, j=1}^{n} \cosh \left(p_{0} p_{j} / r\right) \cosh \left(p_{0} p_{i} / r\right) x_{i} x_{j}+\sum_{i, j=1}^{n} \cosh \left(p_{i} p_{j} / r\right) x_{i} x_{j} \\
=-\sum_{i, j=1}^{n}\left[\cosh \left(p_{0} p_{j} / r\right) \cosh \left(p_{0} p_{i} / r\right)-\cosh \left(p_{i} p_{j} / r\right)\right] x_{i} x_{j} .
\end{aligned}
$$

Thus, $\sum_{i, j=0}^{n} \cosh \left(p_{i} p_{j} / r\right) x_{i} x_{j}$ is negative semidefinite of rank $k$ on the hyperplane $x_{0}=-\sum_{j=1}^{n} \cosh \left(p_{0} p_{j} / r\right) x_{j}$ if and only if $\sum_{i, j=1}^{n}\left[\cosh \left(p_{0} p_{j} / r\right) \cosh \left(p_{0} p_{i} / r\right)-\cosh \left(p_{i} p_{j} / r\right)\right] x_{i} x_{j}$ is positive semidefinite of rank $k$.

3. An application. As an example of the possible utility of the above characterization, we give an affirmative answer to the following problem.

Suppose $p_{0}^{\prime}, p_{1}^{\prime}, \cdots, p_{n}^{\prime}$ and $p_{0}^{\prime \prime}, p_{1}^{\prime \prime}, \cdots, p_{n}^{\prime \prime}$ are vertices of two $n$-simplices in $H_{n, r}$. Let $r_{i j}=r \cosh ^{-1}\left[\cosh \left(p_{i}^{\prime} p_{j}^{\prime} / r\right) \cosh \left(p_{i}^{\prime \prime} p_{j}^{\prime \prime} / r\right)\right]$ $(i, j=0,1,2, \cdots, n)$. Does an $n$-simplex of $H_{n, r}$ exist with edges equal to $r_{i j}$ ?

By the theorem it suffices to consider the quadratic form

$$
K\left(x_{1}, x_{2}, \cdots, x_{n}\right)=\sum_{i, j=1}^{n}\left[\cosh \left(r_{0 i} / r\right) \cosh \left(r_{0 j} / r\right)-\cosh \left(r_{i j} / r\right)\right] x_{i} x_{j} .
$$


Now,

$$
\begin{array}{r}
K\left(x_{1}, x_{2}, \cdots, x_{n}\right) \\
=\sum_{i, j=1}^{n}\left[\cosh \left(p_{0}^{\prime} p_{i}^{\prime} / r\right) \cosh \left(p_{0}^{\prime} p_{j}^{\prime} / r\right) \cosh \left(p_{0}^{\prime \prime} p_{i}^{\prime \prime} / r\right) \cosh \left(p_{0}^{\prime \prime} p_{j}^{\prime \prime} / r\right)\right. \\
\left.-\cosh \left(p_{i}^{\prime} p_{j}^{\prime} / r\right) \cosh \left(p_{i}^{\prime \prime} p_{j}^{\prime \prime} / r\right)\right] x_{i} x_{j} \\
=\sum_{i, j=1}^{n}\left[\cosh \left(p_{0}^{\prime} p_{i}^{\prime} / r\right) \cosh \left(p_{0}^{\prime} p_{j}^{\prime} / r\right) \cosh \left(p_{0}^{\prime \prime} p_{i}^{\prime} / r\right) \cosh \left(p_{0}^{\prime \prime} p_{j}^{\prime \prime} / r\right)\right. \\
-\cosh \left(p_{0}^{\prime \prime} p_{i}^{\prime \prime} / r\right) \cosh \left(p_{0}^{\prime \prime} p_{j}^{\prime \prime} / r\right) \cosh \left(p_{i}^{\prime} p_{j}^{\prime} / r\right) \\
+\cosh \left(p_{0}^{\prime \prime} p_{i}^{\prime \prime} / r\right) \cosh \left(p_{0}^{\prime \prime} p_{j}^{\prime \prime} / r\right) \cosh \left(p_{i}^{\prime} p_{j}^{\prime} / r\right) \\
\left.-\cosh \left(p_{i}^{\prime} p_{j}^{\prime} / r\right) \cosh \left(p_{i}^{\prime \prime} p_{j}^{\prime \prime} / r\right)\right] x_{i} x_{j}
\end{array}
$$

Each quadratic form is positive semidefinite, and hence their sum is also.

\section{REFERENCES}

1. L. M. Blumenthal, Theory and applications of distance geometry, Clarendon Press, Oxford, 1953. MR 14, 1009.

2. - The geometry of a class of semimetric spaces, Tôhoku Math. J. 43 (1937), 205-224.

3. L. M. Blumenthal and G. A. Garrett, Characterization of spherical and pseudospherical sets of points, Amer. J. Math. 53 (1931), 619-640.

4. H. S. M. Coxeter and J. A. Todd, On points with arbitrarily assigned mutual distances, Proc. Cambridge Philos. Soc. 30 (1934), 1-3.

5. K. Menger, Bericht iiber metrische Geometrie, Jber. Deutsch. Math.-Verein 40 (1931), 201-219.

6. - New foundations of euclidean geometry, Amer. J. Math. 53 (1931), 721-745.

7. I. J. Schoenberg, Remarks to Maurice Fréchet's article "Sur la definition axiomatique d'une classe d'espaces distancies vectoriellement applicable sur l'espace de Hilbert," Ann. of Math. (2) 36 (1935), 724-732.

8. - Metric spaces and positive definite functions, Trans. Amer. Math. Soc. 44 (1938), 522-536.

\section{Utah State University}

